\title{
Raman and normal mode studies of the polypeptide chain conformations in crystalline magnesium and calcium poly(L-glutamate) s $^{*}$
}

\author{
P. K. SenguPta and S. KRIMM \\ Biophysics Research Division, The University of Michigan, Ann Arbor, MI 48109, U.S.A.
}

(Received 7 August 1984)

\begin{abstract}
An analysis of the Raman spectra of dry crystalline magnesium and calcium poly(L-glutamate)s, based on a normal mode calculation of $\alpha$-helical poly (L-glutamate), shows that the polypeptide chains in both salts have an $\alpha$-helix conformation. In the wet crystalline state, another chain conformation is also present for the calcium salt.
\end{abstract}

\section{INTRODUCTION}

KEITH et al. [1] have shown that, under appropriate conditions, alkaline earth salts of poly(L-glutamic acid) $\left((\mathrm{GluH})_{n}\right)$ can form lamellar, chain-folded single crystals. The mode of crystal packing, and the conformation adopted by the polypeptide chain, are determined primarily by the nature of the alkaline earth cation and the temperature at which crystallization occurs. We have recently reported a detailed vibrational spectroscopic analysis on the $\beta$-sheet forms of the $\mathrm{Ca}^{2+}$ salt $\left(\beta-(\mathrm{GluCa})_{n}\right)$ [2]. In addition to confirming the antiparallel-chain plcated shect structure which had been proposed [1], this work led to considerable insight into the influence of the side chain on main chain modes, and has stimulated our interest in examining other conformations present in different crystalline ferms of $(\mathrm{GluH})_{n}$ salts.

It is known $[1,3]$ that, at or below room temperature, both the $\mathrm{Mg}^{2+}$ and $\mathrm{Ca}^{2+}$ salts of $(\mathrm{GluH})_{n}$ crystallize in the hexagonal form, which suggested a helical conformation of the polypeptide chain [3]. Indirect arguments, derived from measurements of the $a$ dimensions in X-ray powder diffraction patterns of wet as well as dry crystals, led to proposals for an $\alpha$ helix conformation for the $\mathrm{Mg}^{2+}$ salt [1], and a possible "extended-helix" structure for the $\mathrm{Ca}^{2+}$ salt [3]. The latter was based on earlier suggestions $[4,5]$ that in polypeptides with charged side chains there should be local regions in which the main chain adopts an ordered conformation similar to the left-handed $33_{1}$-helix of poly(L-proline)II. However, since the $c$-axis (fiber axis) repeat distance of these crystals could not be measured, there was no direct crystallographic evidence for either of these proposals.

In the present study, we have used Raman spectroscopy to examine the chain conformations existing in

*This is paper No. 29 in a series, "Vibrational analysis of peptides, polypeptides, and proteins". It is dedicated to Sir HAROLD W. THOMPSON, a colleague whose professional contributions and warm personal friendship will be long remembered. hexagonal crystals of the $\mathrm{Mg}^{2+}$ and $\mathrm{Ca}^{2+}$ salts of $(\mathrm{GluH})_{n}$. Using the force field recently refined for $\alpha$ helical poly(L-alanine) $\left(\alpha-(\mathrm{Ala})_{n}\right)[6]$, we have calculated normal modes for a standard $\alpha$-helical structure of (GluH $)_{n}[6]$, assuming a fully extended side chain as in $\beta$-(GluCa $)_{n}[1]$. These theoretical results, together with our recent work on $\beta$-(GluCa $)_{n}$, have provided a reliable basis for the interpretation of the observed Raman spectra.

\section{EXPERIMENTAL}

Lamellar, hexagonal crystals of (GluMg) and (GluCa) were obtained by following published procedures $[1,3]$, which involved dropwise addition of ethanol to a mixture of aqucous solutions containing the $\mathrm{Na}^{+}$salt of $(\mathrm{GluH})_{n}$ and $\mathrm{Mg}^{2+}$ - or $\mathrm{Ca}^{2+}$-acetate. To prepare the $N$-deuterated derivative, $\mathrm{H}_{2} \mathrm{O}$ and ethanol were replaced by $\mathrm{D}_{2} \mathrm{O}$ and ethanol$D$, respectively. The crystals were dried from slurries in ethanol (or ethanol-D), and Raman spectra were recorded using a spectrometer described previously [2]. The sample of (GluNa) (MW 60,000, DP 380, Lot No. 34F-5011) was obtained from Sigma Chemicals, and $\mathrm{Mg}^{2+}$ - and $\mathrm{Ca}^{2+}$. acetate, reagent grade, were supplied by J. T. Baker and Allied Chemical, respectively.

The details of the normal mode calculations for $\alpha$-helical $(\mathrm{GluH})_{n}$ will be presented elsewhere (P. K. SENGUPTA and S. KRIMM, to be published.)

\section{RESULTS AND DISCUSSION}

Raman spectra of crystalline (GluMg) and its $N$ deuterated derivative are shown in Fig. 1A and B, respectively. The corresponding spectrum for (GluCa) is given in Fig. 1C. Table 1 lists the observed frequencies for the principal conformationally sensitive bands of $(\mathrm{GluMg})_{n}$ and $(\mathrm{GluCa})_{n}$, together with our corresponding calculated frequencies for an $\alpha$-helical structure.

The amide A mode (NH stretch perturbed by Fermi resonance [7]) for (GluMg) is observed as a weak, broad band with a maximum at $\sim 3311 \mathrm{~cm}^{-1}$. This value is indicative of a hydrogen-bonded NH group, and is comparable to that of other $\alpha$-helical polypeptides [7]. In $\beta$-(GluCa $)_{n}$, amide $A$ occurs at $3274 \mathrm{~cm}^{-1}$, the lower frequency being consistent with 


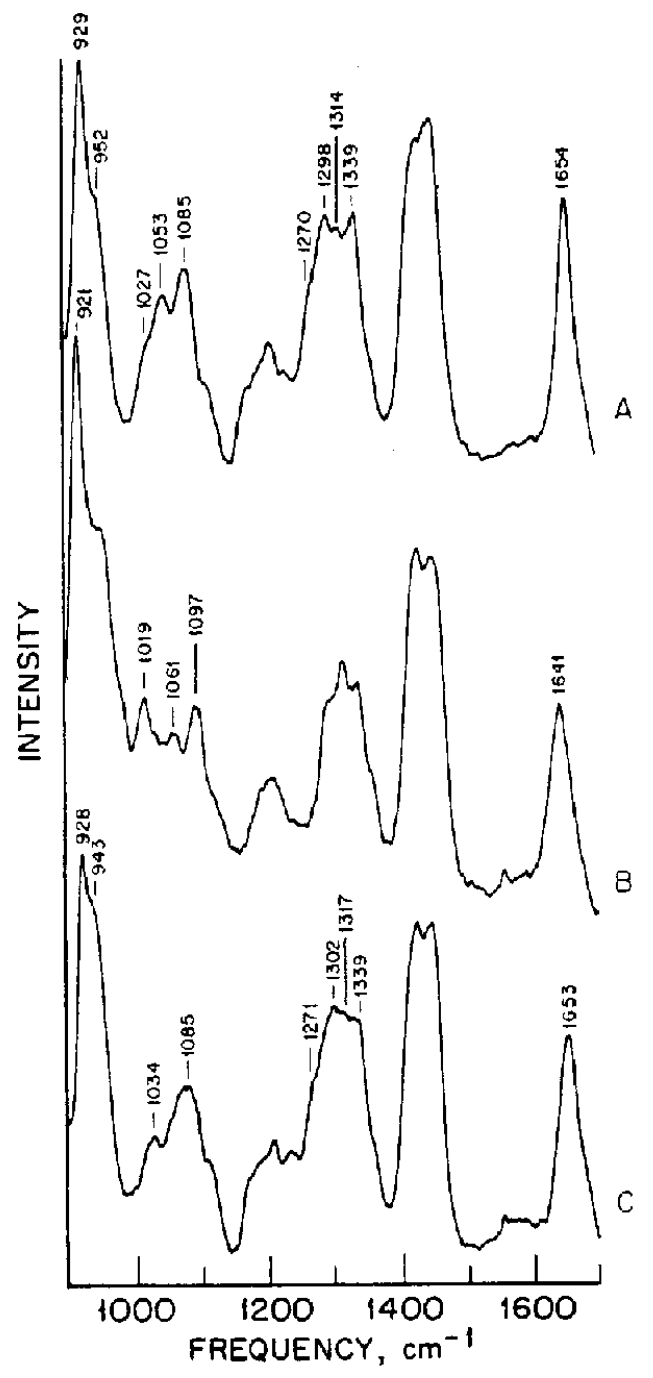

Fig. 1. Raman spectra of dry (A) crystalline (GluMg) crystalline $N$-deuterated (GluMg), (C) crystalline (GluCa) $\left(\lambda_{\text {exc }}=5145 \mathrm{~A}\right.$, spectral slit width $\sim 5.5 \mathrm{~cm}^{-1}$, laser power $80 \mathrm{~mW}$ for (A) and (B), $60 \mathrm{~mW}$ for (C).)

Tabie 1. Observed and calculated frequencies (in $\mathrm{cm}^{-1}$ ) for conformationally sensitive Raman bands of (GluMg $)_{n}$ and (GluCa),

\begin{tabular}{lccl}
\hline & \multicolumn{4}{c}{ Observed } & \\
Mode & $($ GluMg) & $(\text { GluCa })_{n}$ & \multicolumn{1}{c}{ Calculated* } \\
\hline Amide $A$ & $\sim 3311 \mathrm{~W}$ & $\sim 3309 \mathrm{~W}$ & $3279\left(A, E_{1}, E_{2}\right)^{+}$ \\
Amide I & $1654 \mathrm{~S}$ & $1653 \mathrm{~S}$ & $1657(A)$ \\
Amide III & $1339 \mathrm{M}$ & $1339 \mathrm{M}$ & $1343-1326 \ddagger$ \\
& $1298 \mathrm{M}$ & $1302 \mathrm{M}$ & $1299\left(E_{2}\right)$ \\
$1287\left(E_{1}\right)$ \\
& $\sim 1270 \mathrm{sh}$ & $\sim 1271 \mathrm{sh}$ & $1263(A)$ \\
Skeletal & $929 \mathrm{VS}$ & $928 \mathrm{VS}$ & $922(A)$ \\
stretch & & $\sim 943 \mathrm{sh}$ & $\S$ \\
\hline
\end{tabular}

*For standard $\alpha$-helix with fully extended side chain (P. K. Sengupta and S. KrImM, to be published).

$+\mathrm{NH}$ stretch frequency unperturbed by Fermi resonance. $\neq \mathrm{CH}_{2}$ twist $+\mathrm{NH}$ in-plane bend modes (see text). $\$ N$ Non- $\alpha$-helix band (see text) the much stronger hydrogen bond in a $\beta$-sheet structure [2]. The amide I (mainly $\mathrm{C}=\mathrm{O}$ stretch) frequency for (GluMg) appears at $1654 \mathrm{~cm}^{-1}$ in the Raman spectrum, which is identical to the i.r. value [1], and is in the typical range $\left(1650-1657 \mathrm{~cm}^{-1}\right)$ for $\alpha$-helical polypeptides and proteins [8]. In the $N$-deuterated derivative, this mode is found at $1641 \mathrm{~cm}^{-1}$. (Our calculations predict a downshift of $10 \mathrm{~cm}^{-1}$.) Amide III (CN stretch plus NH in-plane bend) generally occurs in the $1200-1300 \mathrm{~cm}^{-1}$ region, and, on the basis of $N$-deuteration studies, we identify this mode with the band at $1298 \mathrm{~cm}^{-1}$. This is very close to the amide III bands of $\alpha-(\mathrm{GluH})_{n}[9]$ and $\alpha$-poly $(\gamma$-benzyl-Lglutamate) [9], which appear in both near $1295 \mathrm{~cm}^{-1}$. In the present case, the observed $1298 \mathrm{~cm}^{-1}$ band can be assigned, by analogy with $\alpha$-(Ala $)_{n}$ [6], to calculated amide III modes at $1287\left(E_{1}\right)$ and $1299\left(E_{2}\right)$, both of which are Raman active. The weak shoulder at $\sim 1270 \mathrm{~cm}^{-1}$ is probably assignable to the calculated $A$ species amide III mode at $1263 \mathrm{~cm}^{-1}$. We note that, in addition to the $1298 \mathrm{~cm}^{-1}$ band, the $1339 \mathrm{~cm}^{-1}$ band also decreases in intensity on $\mathrm{N}$-deuteration. Our calculations predict two kinds of modes in this region: $\mathrm{CH}_{2}$ twist modes at $1343(A), 1341\left(E_{1}\right)$ and $1339\left(E_{2}\right) \mathrm{cm}^{-1}$, and modes at $1326\left(E_{1}, E_{2}\right) \mathrm{cm}^{-1}$ that include significant contributions from $\mathrm{NH}$ in-plane bend. It is likely that the $1339 \mathrm{~cm}^{-1}$ band represents an overlapping of these two kinds of modes. This interpretation was also suggested by KOENIG and SutTon [10] with regard to the $1336 \mathrm{~cm}^{-1}$ Raman band in $\alpha$-poly $(\gamma$-benzyl-L-glutamate).

In addition to the amide I and III modes, a band which occurs in the $900-1000 \mathrm{~cm}^{-1}$ region, and is associated primarily with skeletal stretching vibrations, also seems to be sensitive to the polypeptide backbone conformation $[11,12]$. A strong band in this region is characteristic of the $\alpha$-helix structure [8], and is observed at $908(A) \mathrm{cm}^{-1}$ in $\alpha$-(Ala) $)_{n}[6]$. In the spectrum of (GluMg) $)_{n}$, we observe a very strong band at $929 \mathrm{~cm}^{-1}$, which is in reasonably close agreement with a calculated mode at $922(A) \mathrm{cm}^{-1}$. In $\alpha$ $(\mathrm{GluH})_{n}[9]$ this band appears at $924 \mathrm{~cm}^{-1}$, whereas in $\beta$-(GluCa $)_{n}$ the corresponding frequency is $956 \mathrm{~cm}^{-1}$ [2]. The origin of the shoulder at $\sim 952 \mathrm{~cm}^{-1}$ is not immediately obvious. It may be due to the presence of a small amount of $\beta$-sheet structure, or to some other conformation that occurs on the crystal surface, where the polypeptide chain undergoes folding.

The above results provide strong support for the assignment of an $\alpha$-helix conformation to the polypeptide chain in the hexagonal form of (GluMg) consistent with the conclusions from X-ray studies $[1,3]$.

For crystalline (GluCa) $)_{n}$, the amide $A$, I, III and characteristic skeletal modes are observed at $\sim 3309$, 1653,1302 and $928 \mathrm{~cm}^{-1}$, respectively. These are almost identical to the corresponding frequencies for (GluMg) , the slight differences being well within experimental error. This close agreement between the 
principal Raman bands of crystalline (GluMg) ${ }_{n}$ and $(\text { GluCa })_{n}$ indicates that, at least in the dry state, the polypeptide backbone conformations of these two salts are similar, i.e. $\alpha$-helical. However, we do find differences in widths and relative intensities of some bands. In the $1000-1100 \mathrm{~cm}^{-1}$ region of $(\mathrm{GluMg})_{n}$, we find two distinct peaks at 1053 and $1085 \mathrm{~cm}^{-1}$ (which are in close agreement with calculated values of $1047(A), \quad 1048\left(E_{1}, E_{2}\right)$ and $\left.1085(A) \mathrm{cm}^{-1}\right)$, and a shoulder at $\sim 1027 \mathrm{~cm}^{-1}$. For $(\mathrm{GluCa})_{n}$ these are replaced by a broad band at $\sim 1085 \mathrm{~cm}^{-1}$ plus a peak at $1034 \mathrm{~cm}^{-1}$. This region is dominated by side chain $\mathrm{CC}$ stretch vibrations, and it is possible that some differences exist in the side chain conformations of the two salts. Since the calculations for the extended side chain are in quite good agreement with the observed bands for $(\mathrm{GluMg})_{n}$, we infer the presence of such extended side chains in this salt; this is in agreement with deductions from $\mathrm{X}$-ray diffraction studies $[1,3]$. The dissimilar spectra of (GluCa $)_{n}$ in this region therefore imply that in this salt the side chains are not fully extended. It is interesting that this is one possibility suggested by the smaller $a$-axis dimension of hexagonal (GluCa) [3].

It is also of interest that the $928 \mathrm{~cm}^{-1}$ band of $(\mathrm{GluCa})_{n}$ exhibits a broad shoulder on the high frequency side. In view of the likely sensitivity of this region to backbone conformation $[11,12]$, and the spectroscopic evidence[11-14] in favor of an "extended-helix" conformation $[4,5]$, it is important to consider the possibility that the above shoulder may be associated with a chain conformation other than the $\alpha$-helix. Since solvent can have a strong influence in stabilizing a chain conformation, we examined a slurry of wet crystals of $(\mathrm{GluCa})_{n}$ in a $10 \%$ ethanol-water mixture. (Crystals of (GluMg) were not sufficiently stable in the wet state to obtain spectra.) The Raman spectrum exhibits, in addition to a strong band at $928 \mathrm{~cm}^{-1}$, a medium intensity band at $943 \mathrm{~cm}^{-1}$ (plus a weak shoulder at $\sim 953 \mathrm{~cm}^{-1}$, as in $(\mathrm{GluMg})_{n}$ ). This band seems to be similar to the $\sim 949 \mathrm{~cm}^{-1}$ band of $(\mathrm{GluH})_{n}$ in the charged form $[11,15]$. In a subsequent publication we will consider the relation of this band to the "extended-helix" structure.

In summary, Raman studies of hexagonal (GluMg) and (GluCa), combined with normal mode calculations, establish conclusively that in both salts in the dry state the main chain conformation is $\alpha$-helical, with the side chains being extended in the former case and not in the latter. For (GluCa) ${ }_{n}$ there is evidence for the simultaneous presence of a different backbone conformation, particularly in the wet state.

Acknowledgements-This research was supported by NSF grants PCM-8214064 and DMR-8303610.

\section{REFERENCES}

[1] H. D. Keith, G. Giannoni and F. J. Padden, Biopolymers 1, 775-792 (1969).

[2] P. K. Sengupta, S. Krimm and S. L. Hsu, Biopolymers 23, $1565(1984)$.

[3] H. D. KEITH, Biopolymers 10, 1099-1101 (1970)

[4] M. L. TiffanY and S. KRIMm, Biopolymers 6, 1379-1381 (1968)

[5] S. KRIMM and J. E. Mark, Proc. natn. Acad. Sci. U.S.A. 60, 1122-1129 (1968).

[6] A. M. DWIVEDI and S. KRIMm, Biopolymers 23, 923-943 (1984).

[7] S. KRIMM and A. M. DwIVEDI, J. Raman Spectrosc. 12 , 133-137 (1982).

[8] B. G. Frushour and J. L. Koenig, (1975) in Advances in Infrared and Raman Spectroscopy, Vol. 1, pp. 35-97. Heyden, London.

[9] G. D. FASMAN, K. ItOH, C. S. LIU and R. C. LORD, Biopolymers 17, 1729-1746 (1978).

[10] J. L. KOENIG and P. L. SutTON, Biopolymers 10, 89-106 (1971).

[11] J. L. Koenig and B. Frushouk, Biopolymers 11, 1871-1892 (1972).

[12] T.-J. YU, J. L. Lippert and W. J. Peticolas, Biopolymers 12, 2161-2176 (1973).

[13] W. B. RiPPON and A. G. Walton, J. Am. chem. Soc. 94, 4319-4324 (1972).

[14] P. C. Painter and J. L. Koenig, Biopolymers 15 , 229-240 (1976).

[15] Y. Sugawara, I. Harada, H. Matsuura and T. ShIMANOUCHI, Biopolymers 17, 1405-1421 (1978). 